\title{
Thermo-Mechanical Analysis for an Axisymmetric Functionally Graded Rotating Disc under Linear and Quadratic Thermal Loading
}

\author{
Parth Dinesh Mehta \\ Department of Mathematics, School of Technology, \\ Pandit Deendayal Petroleum University, Gandhinagar, India. \\ E-mail: mehtaparths@gmail.com \\ Manoj Sahni \\ Department of Mathematics, School of Technology, \\ Pandit Deendayal Petroleum University, Gandhinagar, India. \\ Corresponding author: manojsahani117@gmail.com
}

(Received December 5, 2019; Accepted March 9, 2020)

\begin{abstract}
The study presents thermo-mechanical analysis of functionally graded (FG) rotating disc whose material properties, namely, Young's modulus, density and coefficient of thermal expansion in radial direction are tailored from inner to outer radius using power law form. The disc is considered to be under the influence of internal pressure, centrifugal body force and thermal loading of the form linear as well as quadratic. Response of FG disc under linear and quadratic temperature profile subjected to internal pressure as well as centrifugal body force is analysed. An exact solution for stress in radial and tangential directions, under mechanical and thermal loading is presented. Numerical solutions for stresses under internal pressure with uniform thermal loading are obtained using finite element method and its comparison with analytical results is presented graphically. Results for radial displacement, radial stress and tangential stress are depicted graphically and their interpretation has been discussed.
\end{abstract}

Keywords- Functionally graded rotating disc, Stresses, Young's modulus, Coefficient of thermal expansion, Internal pressure.

\section{Introduction}

Functionally graded materials (FGMs) are an advanced class of composite materials with heterogeneous composition of metal and ceramic over the volume of body. Functionally graded materials are made up of metal matrix, containing smooth distribution of ceramic in form of particulates. They are widely used in manufacturing of engineering components, i.e. in air-craft engines, pressure vessels, steam and gas turbine rotors, turbo generators, internal combustion engines, automotive parts such as brake discs and drums, drive shafts, cylinder blocks and pistons etc. Many researchers have taken keen interest in analysis of such innovative material due to its wide area of application. Sayman and Arman (2006) carried out an elastic-plastic stress analysis in a thermoplastic composite disc under a steady state temperature distribution. Kordkheili and Naghdabadi (2007) presented a solution for thermo-elasticity in hollow and solid rotating functionally graded disks. Exact solutions for stresses in functionally graded annular discs rotating at a constant angular velocity under temperature loading (Bayat et al., 2009; Çallığlu, 2011; Çallıŏlu et al., 2011; You et al., 2007) are also found in literature. Bektaş and Akça, (2012) conducted analytical and numerical analysis of FG disc using power law functions. Thawait et al. (2017), used finite element approach and provided linear elastic solution for FG disk with varying thickness. The analysis of this advanced class of materials presented the fact that FGMs have advantage over composite materials as they reduce the magnitude of stresses and strains and also 
provide thermal resistance, as compared to composites (Sahni and Sharma, 2017). Analysis of elastic and creep deformations in FG annular rotating discs and cylinders has also been reported by researchers in the literature. (Sahni and Sahni, 2014, 2015; Sahni et al., 2017). Mehta et al. (2019a) investigated thermal stresses in FG rotating disc with different material gradation cases. Recently, researchers (Paul and Sahni, 2019; Habib et al., 2019; Mehta et al., 2019b) derived solution for FG cylinder with nonlinearly varying material properties using power series method and FE analysis, respectively.

In our study, an analytical and numerical analysis for rotating FG disc under internal pressure and varying thermal load in complete linear and quadratic form is presented.

\section{Mathematical Formulation of Stress Analysis}

The governing differential equation for an axisymmetric rotating FG disc is given as (Çallıoğlu et al., 2011),

$$
r \frac{d \sigma_{r}}{d r}+\sigma_{r}-\sigma_{\theta}+\rho(r) \omega^{2} r^{2}=0
$$

where, $\sigma_{\mathrm{r}}, \sigma_{\theta}, \rho(\mathrm{r})$ and $\omega$ are radial stress, tangential stress, density and angular speed respectively. The solution is derived using stress function $\mathrm{S}(\mathrm{r})$, that satisfies the equation of equilibrium given by eq. (1) and the relation between stresses and stress function is given as,

$$
\sigma_{r}=\frac{S(r)}{r} \text { and } \sigma_{\theta}=\frac{d S(r)}{d r}+\rho(r) \omega^{2} r^{2}
$$

The strain-displacement equation (You et al., 2007) is given as,

$$
\varepsilon_{r}=\frac{d u}{d r} \text { and } \varepsilon_{\theta}=\frac{u}{r}
$$

where, $\varepsilon_{\mathrm{r}}, \varepsilon_{\theta}$ and $\mathrm{u}$ are radial strain, tangential strain and displacement, respectively.

The compatibility condition obtained by eliminating $u$ from above set of eq. (3) is given as,

$$
\varepsilon_{r}=\varepsilon_{\theta}+r \frac{d \varepsilon_{\theta}}{d r}
$$

Using Hooke's law (You et al., 2007), we get the equations for plane stress given as,

$$
\varepsilon_{r}=\frac{1}{Y(r)}\left(\sigma_{r}-v \sigma_{\theta}\right)+\alpha(r) T(r)
$$




$$
\varepsilon_{\theta}=\frac{1}{Y(r)}\left(\sigma_{\theta}-v \sigma_{r}\right)+\alpha(r) T(r)
$$

where, $\mathrm{Y}(\mathrm{r}), \alpha(\mathrm{r}), \mathrm{T}(\mathrm{r})$ and $v$ are Young's Modulus, coefficient of thermal expansion, temperature, Poisson's ratio, respectively. Using eq. (2), (5) and (6) in eq. (4), we obtain,

$$
\begin{aligned}
& r^{2} \frac{d^{2} S}{d r^{2}}+r \frac{d S}{d r}\left(1-r \frac{Y^{\prime}(r)}{Y(r)}\right)+S\left(v r \frac{Y^{\prime}(r)}{Y(r)}-1\right)=-\rho(r) \omega^{2} r^{3}\left(3+v-r \frac{Y^{\prime}(r)}{Y(r)}\right) \\
& -\rho^{\prime}(r) \omega^{2} r^{4}-Y(r) r^{2} \alpha^{\prime}(r) T(r)-r^{2} Y(r) \alpha(r) T^{\prime}(r)
\end{aligned}
$$

Let internal $\left(\mathrm{r}_{\mathrm{i}}\right)$ and external $\left(\mathrm{r}_{\mathrm{o}}\right)$ radii of disc be $20 \mathrm{~cm}$ and $50 \mathrm{~cm}$, respectively. The material properties of FG disc varying in radial direction are defined as,

$$
\begin{aligned}
& Y(r)=Y_{o}\left(\frac{r}{r_{o}}\right)^{t_{1}} \\
& \rho(r)=\rho_{o}\left(\frac{r}{r_{o}}\right)^{t_{2}} \\
& \alpha(r)=\alpha_{o}\left(\frac{r}{r_{o}}\right)^{t_{3}}
\end{aligned}
$$

where, $\mathrm{t}_{1}=0.5194, \mathrm{t}_{2}=-0.4873, \mathrm{t}_{3}=0.5236$ are material gradient parameters. Also, $\mathrm{Y}_{\mathrm{o}}=150 \mathrm{GPa}$, $\rho_{\mathrm{o}}=5.6 \mathrm{~g} / \mathrm{cm}^{3}$ and $\alpha_{\mathrm{o}}=23 \times 10^{-6} 1 /{ }^{\circ} \mathrm{C}$ are Young's modulus, density and coefficient of thermal expansion at external radius, respectively. Figure 1 shows that decrease in Young's modulus and increase in coefficient of thermal expansion facilitates decrease in density. Thermal loading $\mathrm{T}(\mathrm{r})$ in radial direction of FG disc is defined as,

$T(r)=T_{r_{i}}-T_{r e f}+\left(T_{r_{o}}-T_{r_{i}}\right)\left(\left(\frac{r-r_{i}}{r_{o}-r_{i}}\right)^{k_{1}}+\left(\frac{r-r_{i}}{r_{o}-r_{i}}\right)^{k_{2}}\right)$

where, $\mathrm{T}_{\text {ref }}, \mathrm{T}_{\mathrm{r}_{\mathrm{i}}}$ and $\mathrm{T}_{\mathrm{r}_{\mathrm{o}}}$ are reference, inner and outer temperature, respectively. Also, $\mathrm{k}_{1}$ and $\mathrm{k}_{2}$ are temperature gradient parameters, respectively. Here, as depicted in Figure 2, temperature distribution is of complete linear form when $\mathrm{k}_{1}=0$ and $\mathrm{k}_{2}=1$. On considering $\mathrm{k}_{1}=1$ and $\mathrm{k}_{2}=2$, a complete quadratic thermal profile can be obtained. In our study, both increasing and decreasing behaviour of thermal profile is considered. Also, FG disc is under internal pressure given as below: 
International Journal of Mathematical, Engineering and Management Sciences

Vol. 5, No. 4, 744-757, 2020

https://doi.org/10.33889/IJMEMS.2020.5.4.059

$$
\sigma_{r}\left(r_{i}\right)=-q_{i} \text { and } \sigma_{r}\left(r_{o}\right)=-q_{o}
$$

Analysis for thermal loading in radial direction for FG rotating disc is conducted for below cases:

Case 1: $\mathrm{k}_{1}=0$ and $\mathrm{k}_{2}=1$. Using eq. (8)-(11) in eq. (7), we get the stress function $\mathrm{S}(\mathrm{r})$ as,

$$
S(r)=C_{1} r^{\frac{t_{1}+m}{2}}+C_{2} r^{\frac{t_{1}-m}{2}}+C_{3} r^{t_{2}+3}+C_{4} r^{t_{1}+t_{3}+1}+C_{5} r^{t_{1}+t_{3}+2}
$$

where, $\mathrm{C}_{1}$ and $\mathrm{C}_{2}$ are arbitrary constants. The other constants $\mathrm{C}_{3}, \mathrm{C}_{4}$ and $\mathrm{C}_{5}$ are defined as,

$$
\begin{aligned}
& C_{3}=-\frac{\rho_{o} \omega^{2}\left(3+v-t_{1}+t_{2}\right)}{r_{o}^{t_{2}}\left(t_{2}^{2}+6 t_{2}-t_{1} t_{3}-3 t_{1}+v t_{1}+8\right)}, \\
& C_{4}=-\frac{Y_{o} \alpha_{o} t_{3}}{r_{o}^{t_{1}+t_{3}}\left(t_{3}^{2}+t_{1} t_{3}+2 t_{3}+t_{1}+v t_{1}\right)}\left(T_{i}-T_{r e f}+\left(r_{o}-2 r_{i}\right) \frac{T_{r_{o}}-T_{r_{i}}}{r_{o}-r_{i}}\right), \\
& C_{5}=-\frac{Y_{o} \alpha_{o}\left(1+t_{3}\right)}{r_{o}^{t_{1}+t_{3}}\left(t_{3}^{2}+t_{1} t_{3}+4 t_{3}+2 t_{1}+v t_{1}+3\right)}\left(\frac{T_{r_{o}}-T_{r_{i}}}{r_{o}-r_{i}}\right) .
\end{aligned}
$$

From equation (2), radial stress $\sigma_{\mathrm{r}}$ can be obtained as,

$\sigma_{r}(r)=C_{1} r^{\frac{t_{1}+m-2}{2}}+C_{2} r^{\frac{t_{1}-m-2}{2}}+C_{3} r^{t_{2}+2}+C_{4} r^{t_{1}+t_{3}}+C_{5} r^{t_{1}+t_{3}+1}$

The arbitrary constants $\mathrm{C}_{1}$ and $\mathrm{C}_{2}$ are calculated using equation (12) given as,

$$
C_{1}=\frac{\beta_{1}}{y_{1}}-\frac{\beta_{2} y_{1} y_{2}-\beta_{1} z_{1} y_{2}}{y_{1}^{2} z_{2}-z_{1} y_{1} y_{2}} \text { and } C_{2}=\frac{\beta_{2} y_{1}-\beta_{1} z_{1}}{y_{1} z_{2}-z_{1} y_{2}}
$$

where, $\beta_{1}=-q_{i}-C_{3} r_{i}^{t_{2}+3}-C_{4} r_{i}^{t_{1}+t_{3}}-C_{5} r_{i}^{t_{1}+t_{3}+1}, \beta_{2}=-q_{o}-C_{3} r_{o}^{t_{2}+2}-C_{4} r_{o}^{t_{1}+t_{3}}-C_{5} r_{o}^{t_{1}+t_{3}+1}$.

Also, $y_{1}=r_{i}^{\frac{t_{1}+m-2}{2}}$ and $y_{2}=r_{i}^{\frac{t_{1}-m-2}{2}}, z_{1}=r_{o}^{\frac{t_{1}+m-2}{2}}, \mathrm{z}_{2}=r_{o}^{\frac{t_{1}-m-2}{2}}$ and $m=\sqrt{t_{1}^{2}-4 v t_{1}+4}$.

From equation (2), tangential stress $\sigma_{\theta}$ can be obtained as, 


$$
\begin{aligned}
\sigma_{\theta}(r) & =C_{1}\left(\frac{t_{1}+m}{2}\right) r^{\frac{t_{1}+m-2}{2}}+C_{2}\left(\frac{t_{1}-m}{2}\right) r^{\frac{t_{1}-m-2}{2}}+C_{3}\left(t_{2}+3\right) r^{t_{2}+2} \\
& +C_{4}\left(t_{1}+t_{3}+1\right) r^{t_{1}+t_{3}}+C_{5}\left(t_{1}+t_{3}+2\right) r^{t_{1}+t_{3}+1}+\frac{\rho_{o} \omega^{2}}{r_{o}^{t_{2}}} r^{t_{2}+2}
\end{aligned}
$$

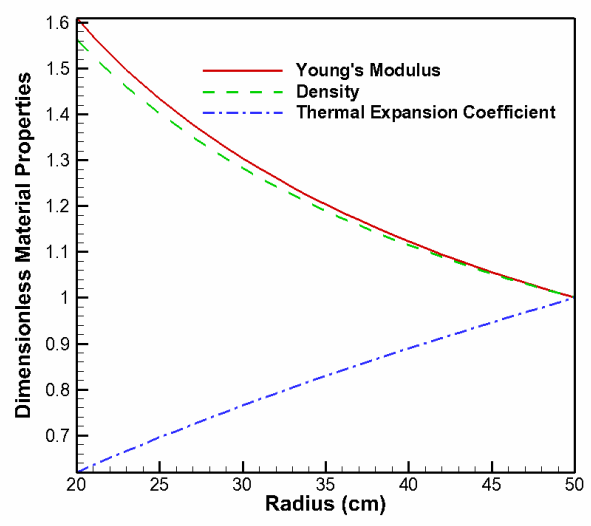

Figure 1. Material properties of FG disc

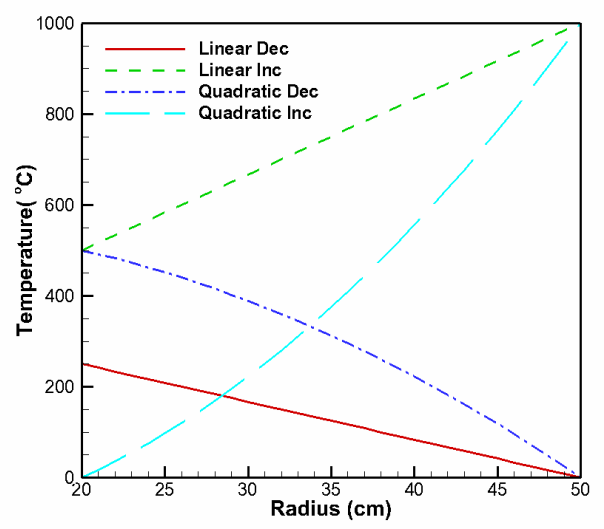

Figure 2. Temperature distribution for FG disc

Case 2: $\mathrm{k}_{1}=1$ and $\mathrm{k}_{2}=2$. Considering the quadratic temperature profile, radial stress can be obtained as,

$\sigma_{r}(r)=C_{1} r^{\frac{t_{1}+m-2}{2}}+C_{2} r^{\frac{t_{1}-m-2}{2}}+C_{3} r^{t_{2}+2}+C_{4} r^{t_{1}+t_{3}}+C_{5} r^{t_{1}+t_{3}+1}+C_{6} r^{t_{1}+t_{3}+2}$

Here, $\mathrm{C}_{1}$ and $\mathrm{C}_{2}$ are constants of integration obtained from boundary condition eq. (12) given as,

$C_{1}=\frac{\beta_{1}}{y_{1}}-\frac{\beta_{2} y_{1} y_{2}-\beta_{1} z_{1} y_{2}}{y_{1}^{2} z_{2}-z_{1} y_{1} y_{2}}$ and $C_{2}=\frac{\beta_{2} y_{1}-\beta_{1} z_{1}}{y_{1} z_{2}-z_{1} y_{2}}$

where, $\beta_{1}=-q_{i}-C_{3} r_{i}^{t_{2}+3}-C_{4} r_{i}^{t_{1}+t_{3}}-C_{5} r_{i}^{t_{1}+t_{3}+1}-C_{6} r_{i}^{t_{1}+t_{3}+2}$,

$\beta_{2}=-q_{o}-C_{3} r_{o}^{t_{2}+2}-C_{4} r_{o}^{t_{1}+t_{3}}-C_{5} r_{o}^{t_{1}+t_{3}+1}-C_{5} r_{o}^{t_{1}+t_{3}+2}$

$y_{1}=r_{i}^{\frac{t_{1}+m-2}{2}}, y_{2}=r_{i}^{\frac{t_{1}-m-2}{2}}, z_{1}=r_{o}^{\frac{t_{1}+m-2}{2}}, \mathrm{z}_{2}=r_{o}^{\frac{t_{1}-m-2}{2}}$ and $m=\sqrt{t_{1}^{2}-4 v t_{1}+4}$. 
The other constants $\mathrm{C}_{3}, \mathrm{C}_{4}, \mathrm{C}_{5}$ and $\mathrm{C}_{6}$ are given as, $C_{3}=-\frac{\rho_{o} \omega^{2}\left(3+v-t_{1}+t_{2}\right)}{r_{o}^{t_{2}}\left(t_{2}^{2}+6 t_{2}-t_{1} t_{3}-3 t_{1}+v t_{1}+8\right)}$,

$$
\begin{aligned}
& C_{4}=-\frac{Y_{o} \alpha_{o} t_{3}}{r_{o}^{t_{1}+t_{3}}\left(t_{3}^{2}+t_{1} t_{3}+2 t_{3}+t_{1}+v t_{1}\right)}\left(T_{i}-T_{r e f}-\left(\frac{T_{r_{o}}-T_{r_{i}}}{r_{o}-r_{i}}\right) r_{i}-\left(\frac{T_{r_{o}}-T_{r_{i}}}{\left(r_{o}-r_{i}\right)^{2}}\right) r_{i}^{2}\right), \\
& C_{5}=-\frac{Y_{o} \alpha_{o} t_{3}}{r_{o}^{t_{1}+t_{3}}\left(t_{3}^{2}+t_{1} t_{3}+4 t_{3}+2 t_{1}+v t_{1}+3\right)}\left(\frac{T_{r_{o}}-T_{r_{i}}}{r_{o}-r_{i}}-2\left(\frac{T_{r_{o}}-T_{r_{i}}}{\left(r_{o}-r_{i}\right)^{2}}\right) r_{i}\right), \\
& C_{6}=-\frac{Y_{o} \alpha_{o} t_{3}}{r_{o}^{t_{1}+t_{3}}\left(t_{3}^{2}+t_{1} t_{3}+6 t_{3}+3 t_{1}+v t_{1}+8\right)}\left(\frac{T_{r_{o}}-T_{r_{i}}}{\left(r_{o}-r_{i}\right)^{2}}\right) .
\end{aligned}
$$

Tangential stress under quadratic thermal loading can be calculated as,

$$
\begin{aligned}
\sigma_{\theta}(r)= & C_{1}\left(\frac{t_{1}+m}{2}\right) r^{\frac{t_{1}+m-2}{2}}+C_{2}\left(\frac{t_{1}-m}{2}\right) r^{\frac{t_{1}-m-2}{2}}+C_{3}\left(t_{2}+3\right) r^{t_{2}+2} \\
& +C_{4}\left(t_{1}+t_{3}+1\right) r^{t_{1}+t_{3}}+C_{5}\left(t_{1}+t_{3}+2\right) r^{t_{1}+t_{3}+1}+C_{6}\left(t_{1}+t_{3}+3\right) r^{t_{1}+t_{3}+2}+\frac{\rho_{o} \omega^{2}}{r_{o}^{t_{2}}} r^{t_{2}+2}
\end{aligned}
$$

From eq. (3) and (6), strains in radial and tangential directions, respectively for linear and quadratic cases of thermal loading can be obtained. In this paper, radial displacement using the same set of equations is presented graphically.

\section{Numerical Computation Scheme}

The governing differential equation (7) coupled with eq. (8) - (11) is solved numerically using finite element method (FEM). The method is implemented using FEM based solver COMSOL Multiphysics (5.4). Considering an axisymmetric geometry of FG disc, discretization of domain is handled using triangular elements of finer size with user-controlled meshing. Thermomechanical analysis of the problem is conducted using solid mechanics module and appropriately defined boundary conditions at inner and outer radius of disc, as given by eq. (12). The model developed is then solved using PARSDISO solver which is a linear direct solver, capable of handling sparse matrices and providing solution of sparse matrices of $A X=b$ form. Results are obtained by applying parametric sweep over appropriate boundary conditions and angular speed for FG rotating disc. The obtained numerical results for radial stress, tangential stress and radial displacement under mechanical and thermal loading follow stiff convergence criterion of $10^{-5}$. In order to obtain accurate results, grid independence test is conducted for grids G1, G2 and G3 consisting of 61746, 762414 and 94438 number of elements, respectively. Figures 3 and 4 depicts radial and tangential 
International Journal of Mathematical, Engineering and Management Sciences

Vol. 5, No. 4, 744-757, 2020

https://doi.org/10.33889/IJMEMS.2020.5.4.059

stress subjected to internal pressure of $\mathrm{q}_{\mathrm{i}}=50 \mathrm{MPa}$ and increasing thermal loading from inner to outer radius for FG disc rotating at $350 \mathrm{rad} / \mathrm{s}$. It can be noted from grid independence test, that radial stress varies only with a difference in values at third decimal place whereas values of tangential stress do not vary at all. Thus, grid G3 has been considered as an appropriate grid for present numerical results. From Figures 3 and 4, it can be observed that numerical results for radial stress and tangential stress are in close agreement with the obtained analytical results. A comparison of some of the obtained exact and numerical results are also shown in the section ahead.

The effect of internal pressure, thermal loading and centrifugal body force on radial displacement and stress in radial as well as tangential directions, for FG disc, is presented graphically. The values used for the parameters are referred from (Çallığlu et al., 2011) in CGS unit system. For increasing thermal loading from inner to outer radius, $\mathrm{T}_{\mathrm{ref}}=0{ }^{\circ} \mathrm{C}, \mathrm{T}_{\mathrm{r}_{\mathrm{i}}}=0{ }^{\circ} \mathrm{C}, \mathrm{T}_{\mathrm{r}_{\mathrm{o}}}=500{ }^{\circ} \mathrm{C}$ and for decreasing thermal loading profile, $\mathrm{T}_{\text {ref }}=0{ }^{\circ} \mathrm{C}, \mathrm{T}_{\mathrm{r}_{\mathrm{i}}}=500{ }^{\circ} \mathrm{C}, \mathrm{T}_{\mathrm{r}_{\mathrm{o}}}=250{ }^{\circ} \mathrm{C}$ are temperature parameters. Also, internal pressure $\mathrm{q}_{\mathrm{i}}=50 \mathrm{MPa}$, angular velocity $\omega=350 \mathrm{rad} / \mathrm{s}$ and $v=0.3$ are considered.

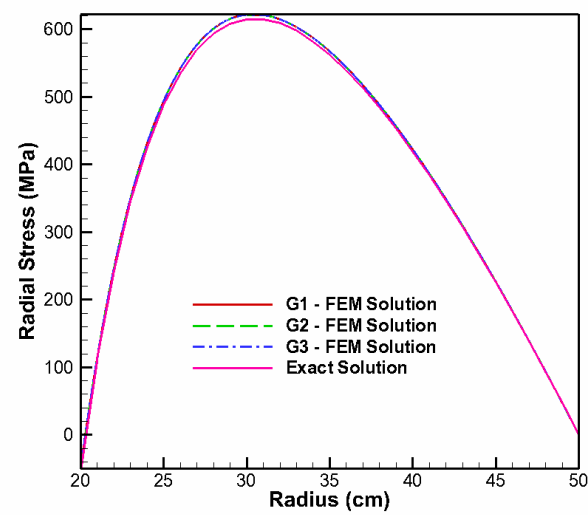

Figure 3. Radial stress under linearly increasing thermal profile, $q_{i}=50 \mathrm{MPa}, T_{r_{i}}=T_{\text {ref }}=0{ }^{\circ} \mathrm{C}$, $T_{r_{o}}=500{ }^{\circ} \mathrm{C}$ and $\omega=350 \mathrm{rad} / \mathrm{s}$

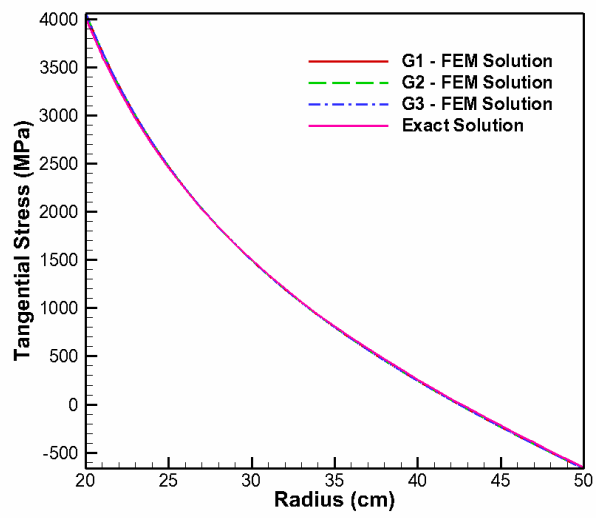

Figure 4. Tangential stress under linearly increasing thermal profile, $\mathrm{q}_{\mathrm{i}}=50 \mathrm{MPa}, \mathrm{T}_{\mathrm{r}_{\mathrm{i}}}=$ $\mathrm{T}_{\text {ref }}=0{ }^{\circ} \mathrm{C}, \mathrm{T}_{\mathrm{r}_{\mathrm{o}}}=500^{\circ} \mathrm{C}$ and $\omega=350 \mathrm{rad} / \mathrm{s}$

\subsection{Analytical Results}

Figures 5 - 18 presents analytical results for radial stress, tangential stress and radial displacement. Figure 5 depicts radial stress under internal pressure of $q_{i}=50,100 \mathrm{MPa}$, for $\mathrm{FG}$ and homogeneous disc. It can be seen from Figure 5 that radial stress is highly compressive at inner radius and tends to decrease in magnitude towards outer radius. From Figure 5, it is evident that radial stress in FG disc has considerably low magnitude than in homogeneous disc. It can be seen from Figure 6, tangential stress under internal pressure, is significantly high at inner radius. This behaviour of tangential stress can be interpreted as the effect of internal pressure at inner radius due to which inner diameter of disc increases causing bursting like effect at inner radial points. Hence, when internal pressure is increased from $50 \mathrm{MPa}$ to $100 \mathrm{MPa}$, it causes further increase in the magnitude 
of tangential stress at inner radial points. Figure 7 presents displacement in disc under internal pressure. It can be seen that displacement in FG disc is lower than in the homogeneous disc and it decreases from inner to outer radius of the disc. Results for increasing thermal loading from inner to outer radius of FG disc under internal pressure and centrifugal body force are presented in Figures $8-10$. Figure 8 represents radial stress in FG disc rotating with angular velocity $\omega=350 \mathrm{rad} / \mathrm{s}$ under internal pressure of $\mathrm{q}_{\mathrm{i}}=50 \mathrm{MPa}$, subjected to linearly and quadratically increasing thermal loading. It is seen that radial stress at inner radius is compressive and has high magnitude but towards the outer radius, it becomes tensile. Also, radial stress under the influence of thermal loading is on the higher side but with introduction of internal pressure, it significantly decreases along the radius of the disc. Importantly, it can be seen that under linear profile radial stress has high magnitude of values whereas under quadratic thermal loading, it decreases. In case of tangential stress as observed from Figure 9, an elastic behaviour is observed under linear and quadratic thermal loading but in linear case, at outer radius, it becomes compressive. Also, under the same loading conditions, displacement as observed from Figure 10 increases from inner to outer radius. Interestingly, the values of displacement are lower at inner radial points under quadratic thermal loading as compared to linear case but ends on higher side at outer radial points. Figures $11-13$ presents result for decreasing thermal loading from inner to outer radius. The radial stress as observed from Figure 11, decreases over the radius of disc when compared to increasing thermal loading. Also, it is interesting to note that radial stress under quadratic thermal loading is on the higher side of magnitude in comparison to linear thermal loading. It is again noted that presence of internal pressure decreases the magnitude of radial stress over the radius of disc. In case of tangential stress, Figure 12 shows that overall magnitude decreases significantly in case of decreasing thermal loading profile. Tangential stress under linearly decreasing thermal loading has high magnitude than quadratically decreasing thermal loading at outer radius but low value at inner radius. Figure 13 shows that under quadratic thermal loading, displacement decreases from inner to outer radius in highly non-linear form as compared to linear thermal loading, in which displacement increases from inner to outer radius. Also, in presence of internal pressure, magnitude of displacement decreases considerably in both form of thermal loading. The effect of body force on behaviour of FG disc under internal pressure is shown in Figures $14-18$. It can be seen from Figure 14, radial stress and tangential stress in FG disc subjected to only centrifugal body force increase in magnitude as the angular velocity increases. The magnitude of radial stress with higher value tends to shift towards outer radius, due to increase in angular velocity. In case of displacement, Figure 15 shows that it is tensile at inner radius but becomes compressive towards outer radius. With increase in angular velocity, displacement along the radius of FG disc increases. The effect of angular velocity on FG disc under internal pressure is depicted in Figures $16-18$. It can be seen from Figure 16, effect of body force $\rho(r) \omega^{2} r$ on radial stress under internal pressure changes the behaviour from compressive to tensile at some radial points, with shift in its magnitude towards the middle region of the disc. As observed from Figure 17, tangential stress has tensile behaviour and has significant increase in its magnitude when the angular velocity increases. In case of displacement, it can be seen from Figure 18 that magnitude of radial displacement increases along the radius of FG disc. 
International Journal of Mathematical, Engineering and Management Sciences

Vol. 5, No. 4, 744-757, 2020

https://doi.org/10.33889/IJMEMS.2020.5.4.059

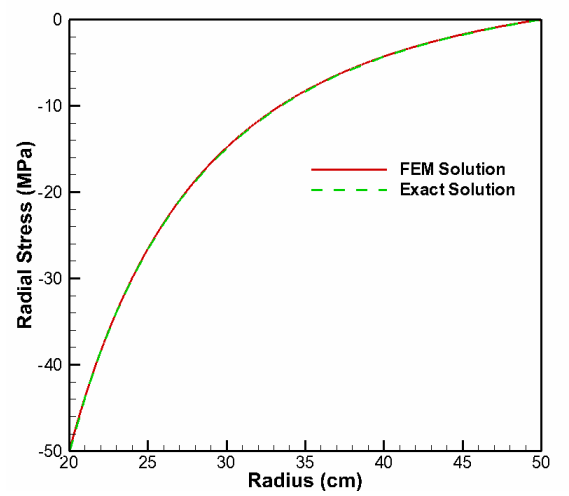

Figure 5. Radial stress under $q_{i}=50,100 \mathrm{MPa}$

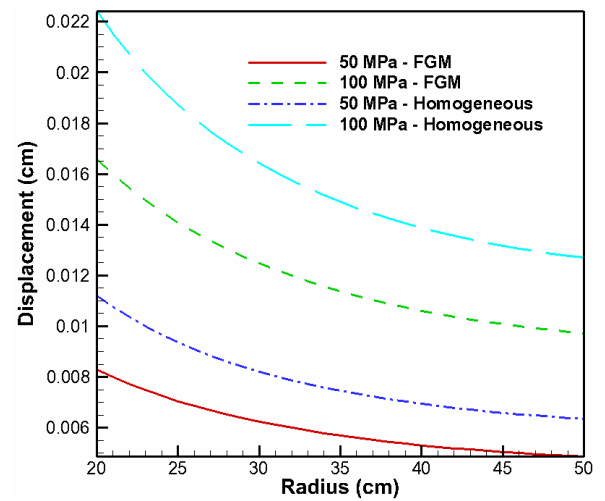

Figure 7. Displacement under $q_{i}=50,100 \mathrm{MPa}$

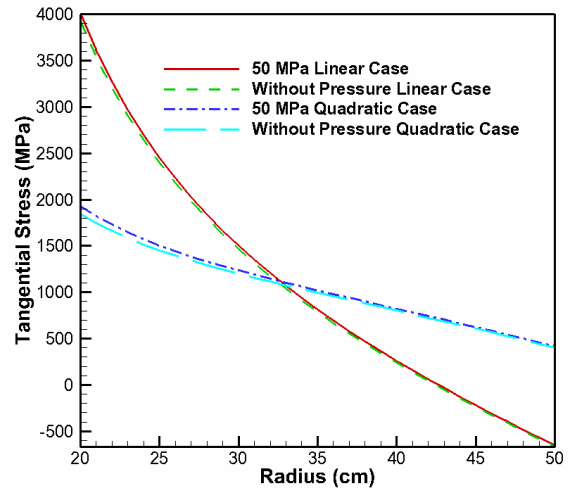

Figure 9. Tangential stress: $q_{i}=50 \mathrm{MPa}, \mathrm{T}_{\mathrm{r}_{\mathrm{i}}}=$ $\mathrm{T}_{\mathrm{ref}}=0{ }^{\circ} \mathrm{C}, \mathrm{T}_{\mathrm{r}_{\mathrm{o}}}=500^{\circ} \mathrm{C}$ and $\omega=350 \mathrm{rad} / \mathrm{s}$

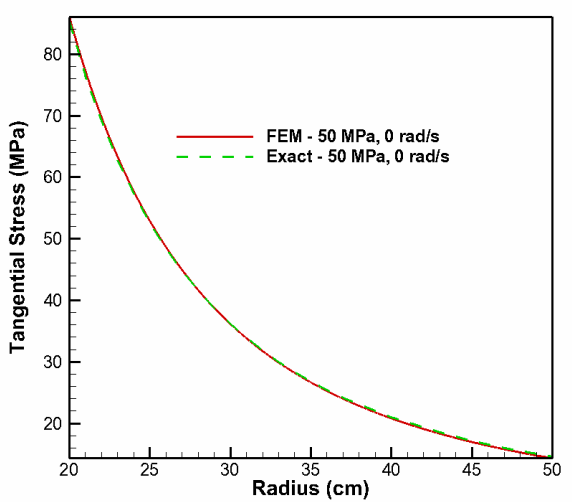

Figure 6. Tangential stress under $q_{i}=50,100$ $\mathrm{MPa}$

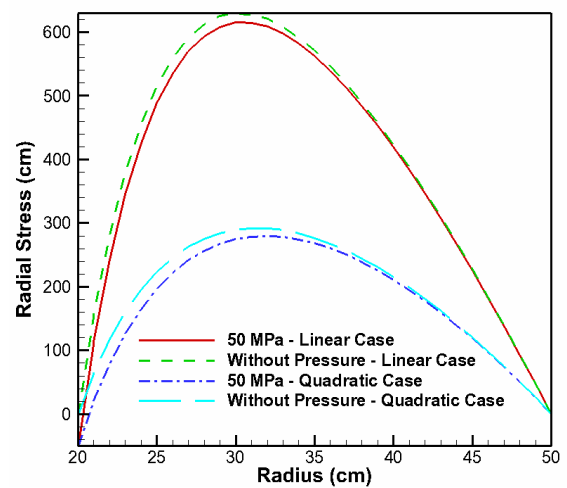

Figure 8. Radial stress: $q_{i}=50 \mathrm{MPa}, T_{r_{i}}=$ $T_{\text {ref }}=0{ }^{\circ} \mathrm{C}, T_{r_{o}}=500^{\circ} \mathrm{C}$ and $\omega=350 \mathrm{rad} / \mathrm{s}$

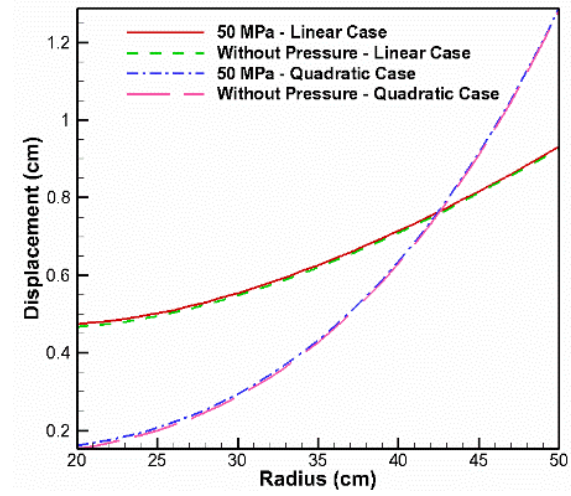

Figure 10. Displacement: $q_{i}=50 \mathrm{MPa}, \mathrm{T}_{\mathrm{r}_{\mathrm{i}}}=$ $\mathrm{T}_{\mathrm{ref}}=0{ }^{\circ} \mathrm{C}, \mathrm{T}_{\mathrm{r}_{\mathrm{o}}}=500^{\circ} \mathrm{C}$ and $\omega=350 \mathrm{rad} / \mathrm{s}$ 
International Journal of Mathematical, Engineering and Management Sciences

Vol. 5, No. 4, 744-757, 2020

https://doi.org/10.33889/IJMEMS.2020.5.4.059

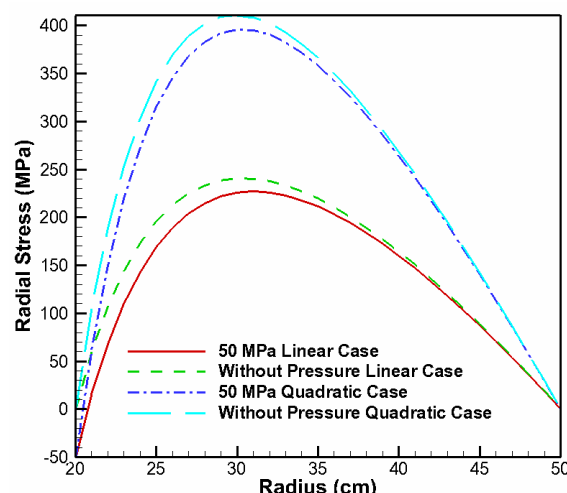

Figure 11. Radial stress: $q_{i}=50 \mathrm{MPa}, T_{r_{i}}=$ $500{ }^{\circ} \mathrm{C}, T_{\text {ref }}=0{ }^{\circ} \mathrm{C}, T_{r_{o}}=250^{\circ} \mathrm{C}$ and $\omega=350$ $\mathrm{rad} / \mathrm{s}$

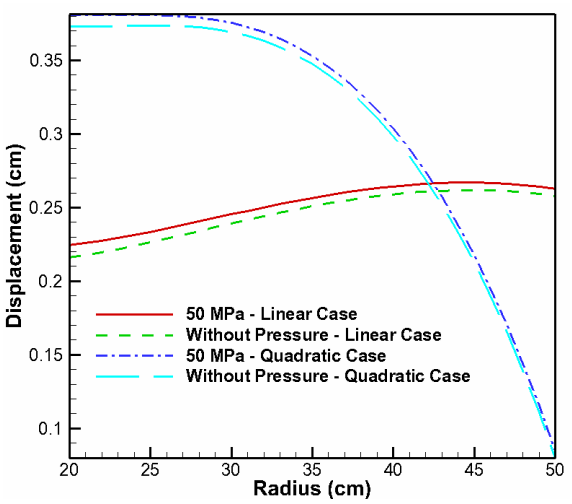

Figure 13. Displacement: $q_{i}=50 \mathrm{MPa}, T_{r_{i}}=$ $500{ }^{\circ} \mathrm{C}, T_{\text {ref }}=0{ }^{\circ} \mathrm{C}, T_{r_{o}}=250{ }^{\circ} \mathrm{C}$ and $\omega=350$ $\mathrm{rad} / \mathrm{s}$

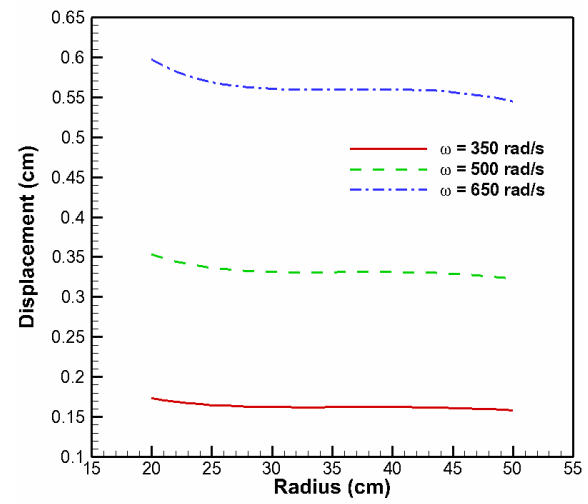

Figure 15. Effect of angular velocity on displacement

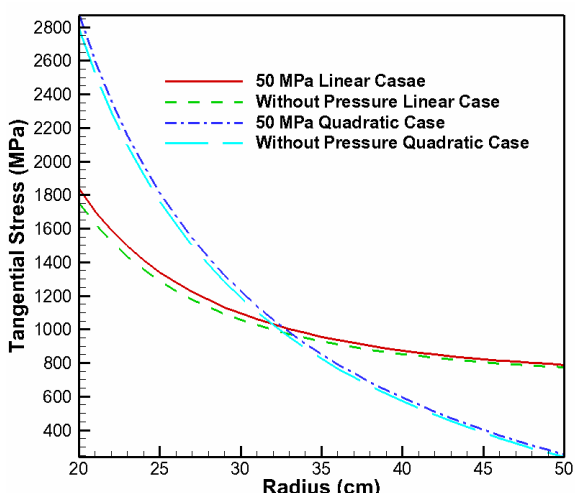

Figure 12. Tangential stress: $q_{i}=50 \mathrm{MPa}, T_{r_{i}}=$ $500{ }^{\circ} \mathrm{C}, T_{\text {ref }}=0{ }^{\circ} \mathrm{C}, \mathrm{T}_{r_{o}}=250^{\circ} \mathrm{C}$ and $\omega=350$ $\mathrm{rad} / \mathrm{s}$

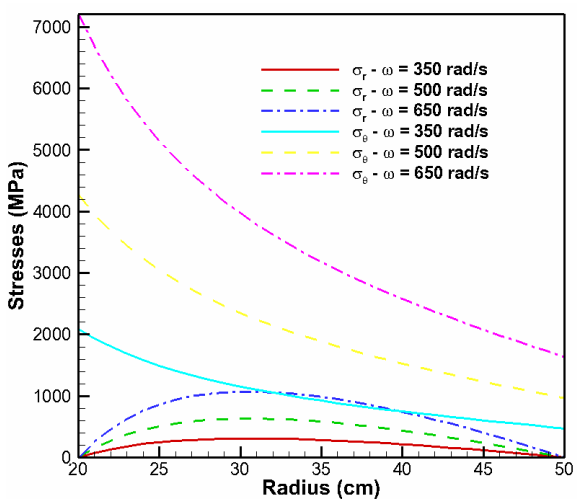

Figure 14. Effect of angular velocity on radial and tangential stresses

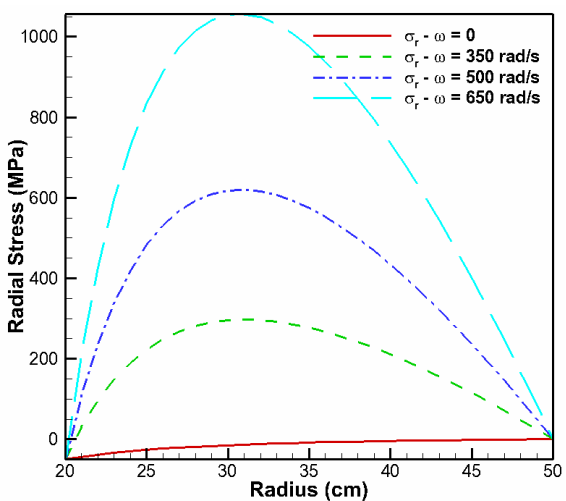

Figure 16. Effect of angular velocity on radial stress under $q_{i}=50 \mathrm{MPa}$ 
International Journal of Mathematical, Engineering and Management Sciences

Vol. 5, No. 4, 744-757, 2020

https://doi.org/10.33889/IJMEMS.2020.5.4.059

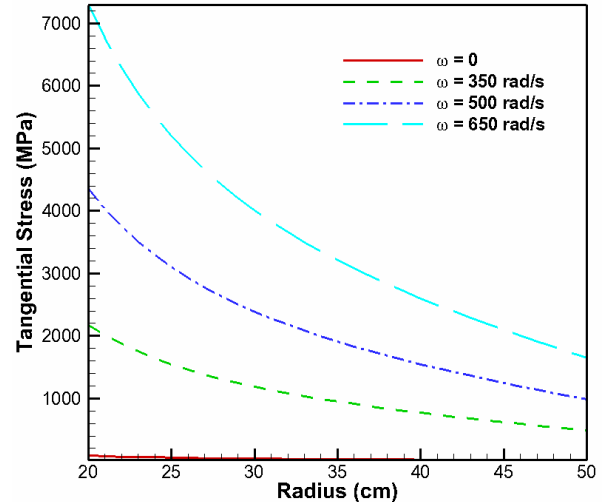

Figure 17. Effect of angular velocity on tangential stress under $q_{i}=50 \mathrm{MPa}$

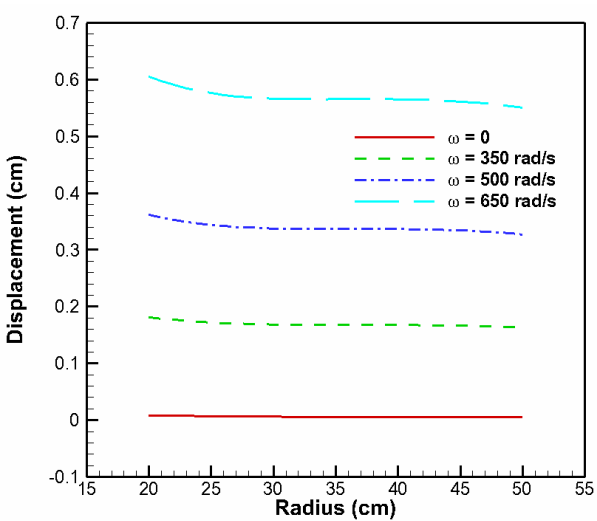

Figure 18. Effect of angular velocity on displacement under $q_{i}=50 \mathrm{MPa}$

\subsection{Comparison of Analytical and Numerical Results}

Figures $19-22$ presents comparison between numerical and analytical results for radial stress and tangential stress in FG disc. Figure 19 presents comparison of exact and numerical solution for radial stress under the influence of internal pressure in FG disc. It is clearly seen that both exact and FEM result are in close agreement, throughout radius of disc. Also, in case of tangential stress, it can be seen from Figure 20 that exact and FEM solutions are consistent with each other. Comparison for an exact and a numerical solution of radial stress under internal pressure and body force with uniform temperature distribution can be seen from Figure 21. It is observed that over the radius of FG disc, both the solutions are in close agreement except around the centre of the disc. For instance, at $r=30 \mathrm{~cm}$ FEM provides the value of $\sigma_{\mathrm{r}}=298.1365 \mathrm{MPa}$ and an exact solution has a value of 295.135 MPa. For tangential stress, both solutions are in agreement as seen in Figure 22.

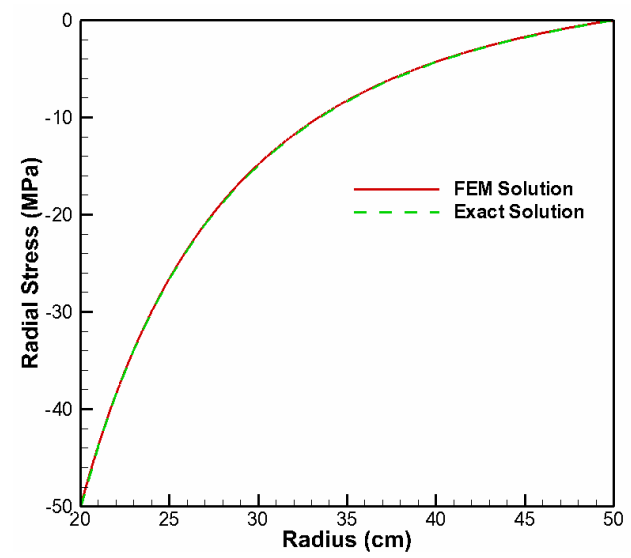

Figure 19. Radial stress under $q_{i}=50 \mathrm{MPa}$

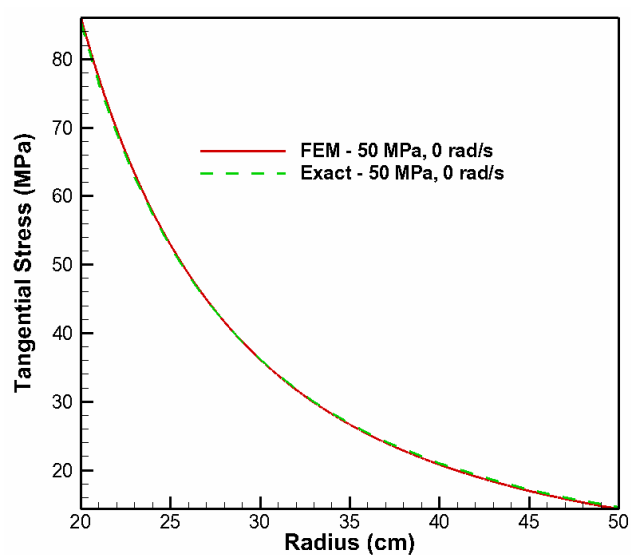

Figure 20. Tangential stress under $q_{i}=50 \mathrm{MPa}$ 


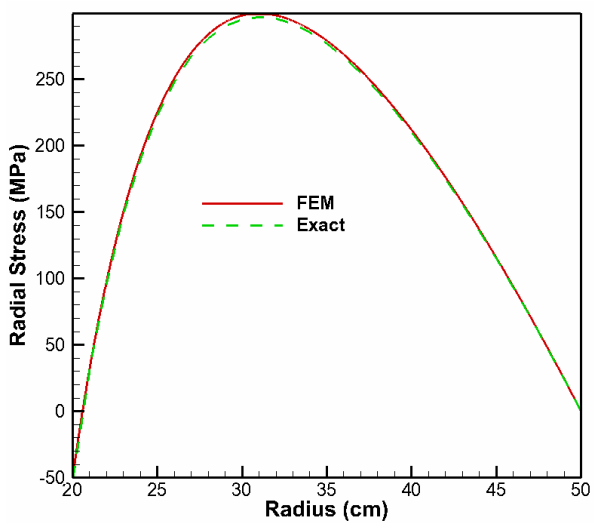

Figure 21. Radial stress: $q_{i}=50 \mathrm{MPa}, T_{r_{i}}=$ $T_{r e f}=T_{r_{o}}=0{ }^{\circ} \mathrm{C}$, and $\omega=350 \mathrm{rad} / \mathrm{s}$

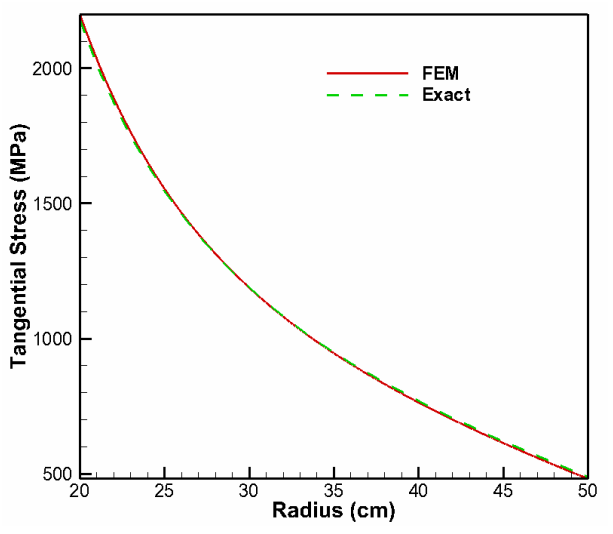

Figure 22. Tangential stress: $q_{i}=50 \mathrm{MPa}, T_{r_{i}}=$ $T_{\text {ref }}=T_{r_{o}}=0{ }^{\circ} \mathrm{C}$, and $\omega=350 \mathrm{rad} / \mathrm{s}$

\section{Conclusion}

In our study, analysis of FG disc subjected internal pressure, thermal loading and body force is conducted. The material properties are varying in power law form from inner to outer radius of cylinder. The effect of varying material properties, effect of thermal loading when the disc is under the influence of pressure and effect of body force is examined. The study leads to some significant observations mentioned as below:

(i) Radial stress in functionally graded disc under the influence of internal pressure, is lower in magnitude when compared with the disc with homogeneous material properties.

(ii) Under the effect of increasing linear thermal loading from inner to outer radius, functionally graded rotating disc subjected to internal pressure has significantly higher magnitude of stress than in the case of quadratic thermal loading but under decreasing thermal distribution from inner to outer radius of the disc, radial stress in quadratic case is higher in magnitude as compared to linear case of thermal distribution.

(iii) Thermo-mechanical radial stress in FG rotating disc under the effect of internal pressure is lower in magnitude as compared to FG disc free from internal pressure.

(iv) Radial stress under the influence of internal pressure increases in magnitude when FG disc is under the effect of centrifugal body force. The increase in angular velocity of FG disc, further increases the magnitude of radial stress.

(v) Tangential stress under the influence of thermomechanical loading has higher magnitude than radial stress at inner radial points which is caused due to the effect of internal pressure.

(vi) Tangential stress under the effect of quadratically increasing thermal distribution, is lower at inner radius and higher at outer radius, as compared to linearly increasing thermal distribution case. The behaviour is reversed when thermal loading decreases from inner to outer radius of $\mathrm{FG}$ disc. 
International Journal of Mathematical, Engineering and Management Sciences

Vol. 5, No. 4, 744-757, 2020

https://doi.org/10.33889/IJMEMS.2020.5.4.059

(vii) Displacement in FG disc under internal pressure decreases from inner to outer radius but with increase in angular velocity, the magnitude of displacement increases.

(viii) Displacement under the effect of increasing thermal loading, internal pressure and body force increases from inner to outer radius of FG disc for both - linear as well quadratic thermal profile. Under decreasing thermal profile, displacement increases from inner to outer radius in linear thermal profile whereas it decreases non-linearly under quadratic thermal profile case. Also, in the presence of internal pressure, displacement under the influence of thermomechanical loading decreases in its magnitude.

\section{Conflict of Interest}

The authors confirm that there is no conflict of interest to declare for this publication.

\section{Acknowledgments}

Authors would like to acknowledge reviewers for their insightful suggestions and valuable comments.

\section{References}

Bayat, M., Saleem, M., Sahari, B.B., Hamouda, A.M.S., \& Mahdi, E. (2009). Mechanical and thermal stresses in a functionally graded rotating disk with variable thickness due to radially symmetry loads. International Journal of Pressure Vessels and Piping, 86(6), 357-372.

Bektaş, N.B., \& Akça, M. (2012). Stress analysis of functionally graded discs under mechanical and thermal loads: analytical and numerical solutions. Science and Engineering of Composite Materials, 19(4), 361371.

Çallıoğlu, H. (2011). Stress analysis in a functionally graded disc under mechanical loads and a steady state temperature distribution. Sadhana, 36(1), 53-64.

Çallığlu, H., Sayer, M., \& Demir, E. (2011). Stress analysis of functionally graded discs under mechanical and thermal loads. Indian Journal of Engineering and Materials Sciences, 18(2), 111-118.

Habib, E.S., El-Hadek, M., \& El-Megharbel, A. (2019). Stress analysis for cylinder made of FGM and subjected to thermo-mechanical loadings. Metals, 9(1), 1-14.

Kordkheili, S.A.H., \& Naghdabadi, R. (2007). Thermoelastic analysis of a functionally graded rotating disk. Composite Structures, 79(4), 508-516.

Mehta, P., Mishra, L., \& Sahni, M. (2019b). Thermo-mechanical stress analysis of thick-walled cylinder with inner FGM layer. Structural Integrity and Life - Integritet I Vek Konstrukcija, 19(3), 211-223.

Mehta, P., Sahni, M., \& Thakur, P. (2019a). Strength analysis of functionally graded rotating disc under variable density and temperature loading. Structural Integrity and Life - Integritet I Vek Konstrukcija, 19(2), 95-101.

Paul S.K., \& Sahni, M. (2019). Two-dimensional mechanical stresses for a pressurized cylinder made of functionally graded material, Structural Integrity and Life - Integritet I Vek Konstrukcija, 19(2), 79-85.

Sahni, M., \& Sahni, R. (2014). Functionally graded rotating disc with internal pressure. Engineering and Automation Problems, 3, 125-130.

Sahni, M., \& Sahni, R. (2015). Rotating functionally graded disc with variable thickness profile and external pressure. Procedia Computer Science, 57, 1249-1254. 
International Journal of Mathematical, Engineering and Management Sciences

Vol. 5, No. 4, 744-757, 2020

https://doi.org/10.33889/IJMEMS.2020.5.4.059

Sahni, M., \& Sharma, S. (2017). Elastic-plastic deformation of a thin rotating solid disk of exponentially varying density. Research on Engineering Structures and Materials, 3(2), 123-133.

Sahni, M., Sahni, R., \& Mehta, P. (2017). Creep behaviour under $\mathrm{SiC}_{\mathrm{p}}$ exponential volume reinforcement in FGM composite rotating cylinders. Materials Today: Proceedings, 4, 9529-9533.

Sayman, O., \& Arman, Y. (2006). Thermal stresses in a thermoplastic composite under a steady state temperature distribution. Journal of Reinforced Plastics and Composites, 25(16), 1709-1722.

Thawait, A., Sondhi, L., Sanyal, S. \& Bhowmick, S. (2017). Elastic analysis of functionally graded variable thickness rotating disk by element based material grading. Journal of Solid Mechanics, 9(3), 650-662.

You, L.H., You, X.Y., Zhang, J.J., \& Li, J. (2007). On rotating circular disks with varying material properties. Zeitschrift für Angewandte Mathematik und Physik, 58(6), 1068-1084. 